\title{
The Lattice Monte Carlo Method for Solving Phenomenological Mass and Thermal Diffusion Problems
}

\author{
Irina V. Belova", Graeme E. Murch", Thomas Fiedler ${ }^{\#}$ and Andreas Öchsner ${ }^{\dagger}$ \\ ${ }^{\#}$ Centre for Mass and Thermal Transport in Engineering Materials, School of Engineering, The \\ University of Newcastle, Callaghan, New South Wales 2308, Australia
} ${ }^{\dagger}$ Department of Applied Mechanics, Faculty of Mechanical Engineering, Technical University of
Malaysia, 81310 UTM Skudai, Johor, Malaysia

Email: Irina.Belova@newcastle.edu.au

\begin{abstract}
In this overview, we introduce the recently developed Lattice Monte Carlo method for addressing and solving phenomenologically-based mass and thermal diffusion problems especially for composite and porous materials. With examples, we describe the application of this numerical method to calculate effective mass diffusivities and concentration profiles. Next, we describe the application of this method to the calculation of effective thermal conductivities/thermal diffusivities and temperature profiles.
\end{abstract}

\section{INTRODUCTION}

The solving of phenomenological mass or thermal diffusion problems is often required in developing many technological processes. When exact solutions are unavailable, such problems are generally solved with finite element or finite-difference numerical methods. This is generally facilitated by the widespread availability of commercial software dedicated to the purpose. However, there are still many occasions when such numerical methods are almost impossible to implement for these problems. Recently, a Lattice Monte Carlo (LMC) method has been intensively developed for addressing phenomenological mass and thermal diffusion problems[1-4]. Although the Monte Carlo method has a reputation for being a computationally very demanding one, this is now much less of a problem as computers have become faster, less expensive and more accessible. The special advantage of the LMC method over the other methods above is that it can be used to address virtually any phenomenological diffusion problem. The LMC method makes use of a very fine-grained lattice that is overlaid on the phenomenological problem. This lattice is then explored by virtual mass or thermal particles to represent mass or thermal diffusion phenomena respectively. In this sense, the LMC method is a form of finite-difference method that is embedded in a quasisimulation of the diffusion process. In this review paper, we trace the history of applications of the LMC method with reference to phenomenological mass and thermal diffusion problems.

As examples of application to mass diffusion problems we discuss the calculation of effective diffusion coefficients in grain boundary diffusion (Harrison Type-A kinetics regime), the effective diffusivity in two-phase systems and the effective ionic conductivity of composite electrolytes. Next, we discuss the determination of concentration profiles for grain boundary diffusion and the segregation of oxygen at interfaces during in-diffusion and out-diffusion of oxygen in cer-mets. For phenomenologically-based thermal diffusion problems, we discuss the calculation of the effective thermal conductivity/diffusivity in some model composites and porous metals and transient temperature profiles in composites. 


\section{MASS DIFFUSION}

The effective mass diffusivity represents the long time limit mass diffusivity in a 'composite material'. This effective diffusivity is probably best known as the diffusivity measured in a tracer diffusion experiment made on a polycrystalline material in the Harrison Type-A kinetics regime[5]. In this regime, the diffusion length of the lattice diffusivity is of the order of the grain size and the effective diffusivity is some weighted average of the lattice diffusivity of the diffusant and the grain boundary diffusivity. Other examples are the effective ionic conductivity of a composite solid electrolyte[6] and the effective diffusivity of a diffusant in a two-phase material[7].

The LMC Method for Determining the Effective Mass Diffusivity. Mass diffusion is a random process that can be represented by random walks of particles. The century-old EinsteinSmoluchowski (ES) Equation[9,10] (often referred to simply as the Einstein Equation) describes the self-diffusivity $D$ of randomly walking particles in $d$ dimensions $(d=1,2,3)$ :

$$
D=\frac{<R^{2}>}{2 d t}
$$

(where $R$ is the vector displacement of a given particle after some long time $t$ and the Dirac brackets $<>$ refer to an average over a very large number $(N)$ of particles).

The ES Equation refers to a system already at equilibrium. In a mass diffusion context, the ES Equation refers then to the diffusivity of individual particles that can be followed or traced in a system that is already at chemical equilibrium, i.e. with no concentration gradient acting or external field(s) acting. Each particle is followed for some long time $t$ in order to determine its displacement $R$ from its original position.

For complete i.e. uncorrelated random walks on a simple cubic lattice the diffusivity can be partitioned from the ES Equation (eq. (1)) as; see, for example, ref [10]:

$$
D=\frac{\Gamma r^{2}}{6}
$$

where $\Gamma$ is the particle jump rate, $r$ is the jump distance (the distance between sites).

It is important to recognize that eq. (1) remains valid for long times even when the material has different diffusivities in different regions of the material (for example in a composite), provided that the material remains isotropic in its diffusion properties overall. The implication also is that each particle 'explores' a sufficiently large portion of the composite structure to be representative of the structure. The diffusivity represented in the ES equation is then the effective diffusivity $D_{\text {eff }}$ of the structure.

By mapping a very fine grained lattice over the composite structure, and keeping the jump distance $r$ the same everywhere (in principle this can be varied however), the diffusivities $D$ in different regions of the structure can be simply represented by correspondingly different jump rates $\Gamma$. It should be noted that since each particle is released independently and diffuses independently there are no correlation effects to be concerned with. Thus these LMC calculations are rather different in concept from atomistic Monte Carlo simulations; see, for example, ref [11]. Because the size of the jump distance $r$ can be varied LMC calculations can be considered as spatially multiscale calculations.

In many mass diffusion problems apart from spatially different diffusivities there may also be segregation of the diffusant between regions of the structure. This is readily represented by different rates in and out of the different regions. Thus if the segregation factor $\mathrm{s}$ between two regions 1 and 2 is defined as:

$$
s=C_{2} / C_{1}
$$


where $C_{1}$ and $C_{2}$ are the concentrations of the diffusant in regions 1 and 2 respectively, then the jump rate $\left(\Gamma_{12}\right)$ into region 1 from region 2 and vice versa $\left(\Gamma_{21}\right)$ are related to s by:

$$
s=\Gamma_{12} / \Gamma_{21}
$$

The simulation of actual time is not realizable in any Monte Carlo kinetics calculation. A discrete quantity that is proportional to actual time is generally used. This quantity is the number of jump attempts per particle during the calculation. Using the ES Equation, with this quantity acting as 'time', one can then calculate a relative diffusivity, i.e. a diffusivity that is relative to one of the specified diffusivities (usually the highest) in the system.

Further details on how these multi-scale modelling LMC calculations are performed can be found in refs 1 and 2. Here, we describe several examples of applications to mass diffusion.

Examples of the Effective Mass Diffusivity Calculated by the LMC method. The effective diffusivity of a diffusant in a material with grain boundaries is very important, especially for describing the rate of penetration of solute in (and out) of the material. As contributions to the effective diffusivity in a microcrystalline or nanocrystalline material, we can identify the diffusivity of the diffusant in the grains: this is the bulk or lattice diffusivity $D_{1}$ and the diffusivity $D_{\mathrm{gb}}$ of the diffusant in the grain boundaries. In the well-known tracer diffusion experiment, a very thin layer of tracer (the diffusant) is deposited at the surface at time $t=0$, the tracer is then permitted to diffuse into the material for some diffusion anneal time $t$. In this scenario it has been traditional to represent grain boundaries as parallel 'slabs' that are normal to the surface'. In the Harrison Type-A kinetics regime, the diffusivity that is determined experimentally from the (Gaussian) tracer concentration depth profile is the effective diffusivity of the material. (In this regime, the tracer diffusion length is of the order of the grain size.) The effective diffusivity is given exactly (for parallel slabs) by the (corrected) Hart-Mortlock Equation[5]:

$$
D_{\text {eff }}=\left(s g D_{g b}+(1-g) D_{l}\right) /(1-g+s g)
$$

where $g$ is the grain boundary volume fraction. For self diffusion $s=1$. However, eq. (5) had never been tested for more realistic grain structures. In Fig. 1 the effective diffusivity as calculated by LMC is shown as a function of grain boundary fraction $g$ for a cubic grain model[12]. It is clear that the Hart-Mortlock Equation does not in fact provide a good description of the effective diffusivity in this situation.

Perhaps surprisingly, the century-old Maxwell-Garnett Equation with the recent correction for possible segregation[13]:

$$
\frac{D_{e f f}}{D_{g b}}=\frac{2(1-g) D_{g b}+(1+2 g) s D_{l}}{(1-g+s g)\left((2+g) D_{g b}+(1-g) s D_{l}\right)}
$$

provides very good agreement with the LMC data, as can be seen in Fig. 1. For other grain models, a similar finding is obtained. For microcrystalline materials the Hart-Mortlock Equation overestimates the actual effective diffusivity by between 10 and $50 \%$ depending on the volume fraction of material in the grain boundaries and the degree of solute segregation. On the other hand, for nanocrystalline materials the use of the Hart-Mortlock equation would overestimate the effective diffusivity by about $45 \%$ and this is largely independent of the degree of solute segregation[14]. The Maxwell-Garnett Equation always does very well in all of the cases that have been examined so far and should therefore routinely replace the Hart-Mortlock Equation. 


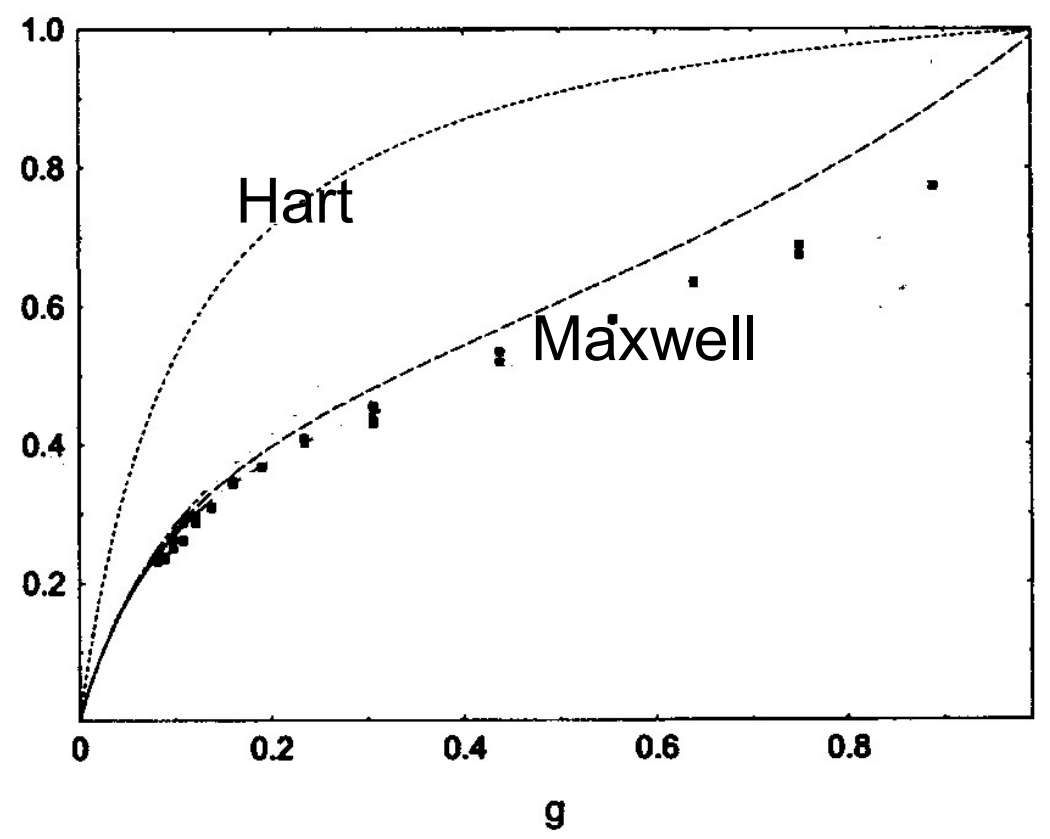

Figure 1. Dependence of the effective diffusivity $D_{\text {eff }} / D_{1}$ on the volume fraction of grain boundaries $g$ for the grain model for a solute segregation factor $s=10$ and $D_{g b} / D_{l}=10^{3}$. Data points are LMC estimates.

Engineering materials are frequently used in multiphase form. Their microstructural stability and resulting longevity at high temperatures are determined by mass transport associated with the interphase boundaries (where the diffusivity is the highest). Addressing this problem requires in the first instance the calculation of the effective diffusivity in a three 'phase' system (the two different grains and the interphase boundary). An equation analogous to the Hart-Mortlock equation can be readily derived for this situation[15]:

$$
\frac{D_{e f f}^{\text {Hart }}}{D_{b}}=k^{\prime}\left(\frac{g_{A} D_{A}}{s_{A} D_{b}}+\frac{g_{B} D_{B}}{s_{B} D_{b}}+g_{b}\right)
$$

where

$$
k^{\prime}=\left(g_{b}+\frac{g_{A}}{s_{A}}+\frac{g_{B}}{s_{B}}\right)^{-1}
$$

and where $D_{\mathrm{A}}$ and $D_{\mathrm{B}}$ are the bulk diffusivities, $g_{\mathrm{A}}$ and $g_{\mathrm{B}}$ are the volume fractions in the two types of grain $A$ and $B$ and $s_{\mathrm{A}}$ and $s_{\mathrm{B}}$ are the corresponding two (in general, different) segregation factors. An equation directly in the spirit of the Maxwell-Garnett Equation for the effective diffusivity has also been derived[15]:

$$
\frac{D_{e f f}}{D_{b}}=k^{\prime} \frac{1-2 g_{\mathrm{A}} \frac{s_{A} D_{b}-D_{A}}{2 s_{A} D_{b}+D_{A}}-2 g_{\mathrm{B}} \frac{s_{B} D_{b}-D_{B}}{2 s_{B} D_{b}+D_{B}}}{\left(1+g_{\mathrm{A}} \frac{s_{A} D_{b}-D_{A}}{2 s_{A} D_{b}+D_{A}}+g_{\mathrm{B}} \frac{s_{B} D_{b}-D_{B}}{2 s_{B} D_{b}+D_{B}}\right)}
$$


and a very useful version of this:

$$
\frac{D_{e f f}}{D_{b A}}=k^{\prime} \frac{1-2 g_{\mathrm{B}} \frac{s_{B} D_{b A}-D_{B}}{2 s_{B} D_{b A}+D_{B}}}{\left(1+g_{\mathrm{B}} \frac{s_{B} D_{b A}-D_{B}}{2 s_{B} D_{b A}+D_{B}}\right)}
$$

Where

$$
\frac{D_{b A}}{D_{b}}=\frac{1-\frac{2 g_{\mathrm{A}}\left(D_{b} s_{A}-D_{A}\right)}{\left(1-g_{B}\right)\left(2 D_{b} s_{A}+D_{A}\right)}}{1+\frac{g_{\mathrm{A}}\left(D_{b} s_{A}-D_{A}\right)}{\left(1-g_{B}\right)\left(2 D_{b} s_{A}+D_{A}\right)}}
$$

For a model of cubic alternating $A$ and $B$ grains, the effective diffusivity has been calculated by the LMC method. eq. (7) was found to be in poor agreement with the results but on the other hand eq. (8) and (9), (10) were shown to describe the effective diffusivity very well[15].

It is well-known that very considerable enhancement of the ionic conductivity of a material is possible by the addition of small, insulating and insoluble particles[6]. In these 'composite electrolytes,' it is believed a highly conducting space-charge layer develops at the interface between the matrix and the insulating particles and this is responsible for the conductivity enhancement. A phenomenological treatment of the problem can shed light on the role of the shape, distribution and size of the insulating particles on the effective conductivity of the material. The ionic conductivity of each of the three 'phases' (matrix, insulating particles, highly conducting layer) is related to the diffusivity of that phase by the Nernst-Einstein Equation:

$$
\sigma_{i}=C_{i} q^{2} D_{i} / k T
$$

where $q$ is the charge on the ion, $C$ is the concentration (of charge carriers), $k$ is the Boltzmann constant and $T$ is the absolute temperature.

A Maxwell-Garnett type equation can be derived to describe the effective ionic conductivity[16]:

$$
\sigma_{e f f}=\frac{\sigma_{2}\left(\left(3-2 g_{2}\right) \sigma_{10}+2 g_{2} \sigma_{2}\right)}{g_{2} \sigma_{10}+\left(3-g_{2}\right) \sigma_{2}}
$$

where

$$
\sigma_{10}=\frac{\sigma_{1}\left(\left(3 g_{2}+g_{1}\right) \sigma_{0}+2 g_{1} \sigma_{1}\right)}{g_{1} \sigma_{0}+\left(3 g_{0}+2 g_{1}\right) \sigma_{1}}
$$

and $\left\{\sigma_{0}, g_{0}\right\},\left\{\sigma_{1}, g_{1}\right\}$ and $\left\{\sigma_{2}, g_{2}\right\}$ are the ionic conductivities and the fractions of the whole occupied by the insulating core (usually $\sigma_{0} \rightarrow 0$ ), highly conducting layer (coating phase) and the matrix respectively. LMC calculations[16] of the effective ionic conductivity show that this equation well describes the enhancement of the effective ionic conductivity for both cubic and spherical insulating particles at relatively low volume fractions of the insulating phase. At higher volume fractions of the insulating particles, the LMC calculations clearly show that the highly conducting coatings of adjacent particles start to touch. This percolation-type behaviour then results 
in a very rapid increase of the ionic conductivity as the percolation threshold is approached. Then there are substantial deviations from the predictions of eq. (12).

The LMC Method for Determining Concentration Profiles. Obtaining the concentration profile using the LMC method is equivalent to solving the Diffusion Equation (Fick's Second Law) for the diffusion problem once initial and boundary conditions have been specified for the problem. The basic LMC procedure to generate concentration profiles has a many similarities to that described already for determining the effective diffusivity. We illustrate the procedure with the well-known tracer diffusion situation mentioned above wherein a very thin layer of tracer (the diffusant) is deposited at the surface at time $t=0$ and then permitted to diffuse into the material for some diffusion anneal time $t$. A source of particles is established from which $N$ particles are released sequentially. Each particle is permitted to diffuse for the (anneal) time $t$, at which time the final position of is recorded. This process is repeated. The final positions of all of the particles are then simply assembled to form a concentration penetration profile. The example presented in Fig. 2 shows the familiar Gaussian profile for tracer diffusion into a semi-infinite solid from a very thin source at the surface $(\mathrm{x}=0)$. Here we plot the concentration ratio $\mathrm{C} / \mathrm{C}_{0}$ as function of the distance from the source, $\mathrm{C}_{0}$ is the initial concentration at the source.

In order to simulate a constant source at the surface, all particles are released simultaneously at $\mathrm{t}=0$ but are still allowed to diffuse completely independently. As each particle leaves the source it is immediately replaced. On the other hand, if a particle returns to the source it is immediately annihilated.

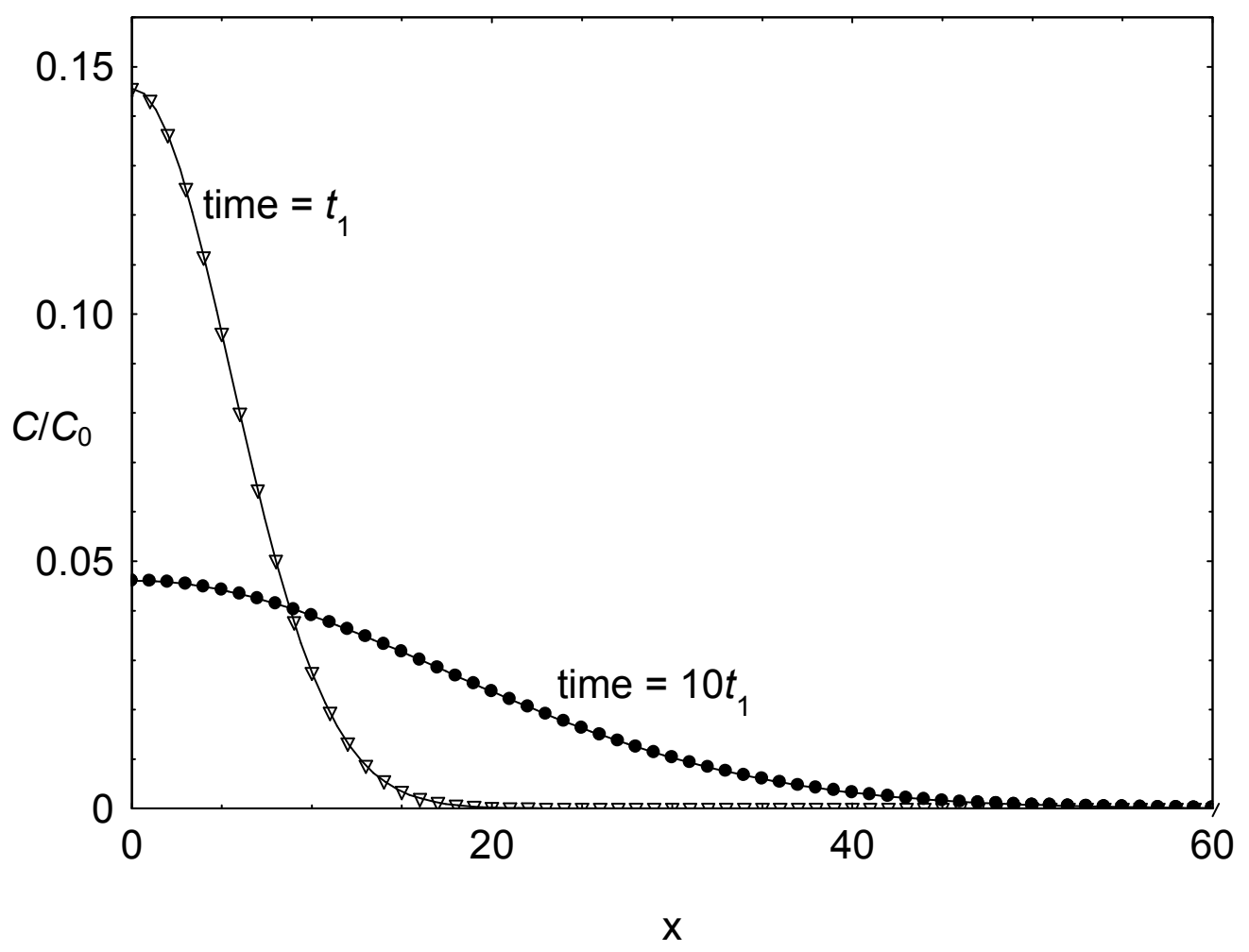

Fig. 2. Typical Gaussian concentration profiles obtained by LMC for diffusion into a semi-infinite solid from a thin-film source at $x=0$ for two different diffusion anneal times.

Examples of Concentration Profiles Calculated by the LMC Method. The determination of concentration profiles in the presence of grain boundaries for diffusion from the thin-film tracer source is of interest to determine the transition between the Harrison Type-A kinetics regime, where the (Gaussian) concentration depth profile provides the effective diffusivity as discussed above, and the Harrison Type-B kinetics regime, where the concentration depth profile has two sections: the 
first section providing $D_{1}$ and the second or tail-section providing $s \delta D_{\mathrm{gb}}$ where $\delta$ is the grain boundary 'width'. Analysis of profiles computed by the LMC method enabled the transition point between the two regimes to be estimated to occur at $\Lambda=L /\left(D_{1} t\right)^{1 / 2}=0.4(L$ is the average spacing between the grain boundaries) for the parallel slabs model, which is much higher then had been estimated previously[17]. Other grain models have also been analyzed with LMC and have shown transition points at $\Lambda=0.4$ for the square grains model and $\Lambda=1.5$ for the cubic grains model[18].

The oxygen that diffuses into metal/metal-oxide cer-mets in-service or during the initial cermet synthesis can strongly segregate to the interfaces between the metal and the metal oxide inclusions. As a consequence of this there can be serious degradation of mechanical properties of the composite. High temperature annealing can remove the oxygen from the cer-met by the process of diffusion-limited evaporation (out-diffusion). Both processes have been modelled by the LMC method[19]. A typical example profile is shown in Fig. 3 for oxygen diffusion into a $\mathrm{Ag} / \mathrm{MgO}$ cermet from a constant oxygen source at the surface.

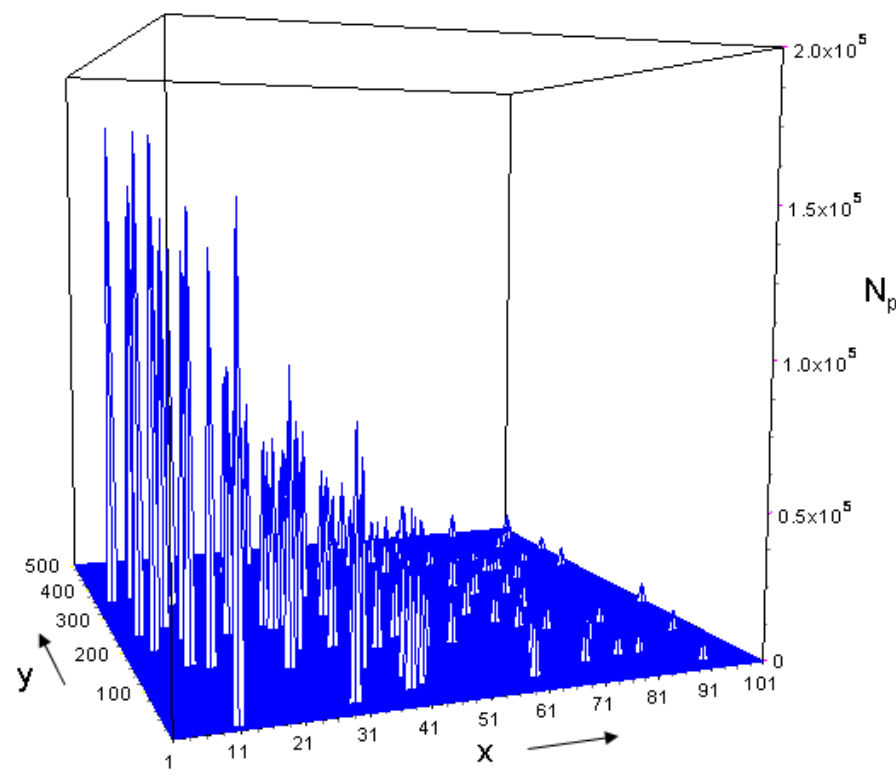

Fig. 3. A typical distribution map of oxygen in a model $\mathrm{Ag} / \mathrm{MgO}$ cer-met with randomly distributed inclusions of $\mathrm{MgO}$ with allowance made for segregation of oxygen at the metal-ceramic interface $(\mathrm{s}=1000)$. The (constant) source of oxygen is at plane $\mathrm{x}=1$ here. Time is 544.2 Monte Carlo time units. $\mathrm{N}_{\mathrm{p}}$ is the number of particles which is linearly related to the oxygen concentration.

\section{THERMAL DIFFUSION}

The LMC Method for Determining the Effective Thermal Diffusivity and Conductivity. Thermal diffusion, like mass diffusion, is a random process that can be represented by random walks of particles. In the case of thermal diffusion, the particles are virtual thermal 'particles'. The ES Equation also describes the thermal diffusivity $K$ in $d$ dimensions $(d=1,2,3)$ :

$$
K=\frac{<R^{2}>}{2 d t}
$$

It should be noted that the thermal conductivity $\lambda_{i}$ in a phase $\mathrm{i}$ is directly related to the thermal diffusivity $K_{\mathrm{i}}$ in that phase by the well-known expression $K_{\mathrm{i}}=\lambda_{\mathrm{i}} / \rho_{i} C_{p, i}$ where $\rho_{i}$ is the density of phase $\mathrm{i}$ and $C_{p, i}$ is its specific heat. It has been shown[19] that for the purposes of a LMC calculation the following procedure of calculating effective thermal conductivity indeed works very well. In a 
model composite, the conductivities $\lambda_{\mathrm{i}}$ are treated in exactly the same way as if they were thermal diffusivities $\left(\lambda_{\mathrm{i}} \Rightarrow K_{\mathrm{i}}^{\prime}\right)$ and calculate effective thermal conductivity $\lambda_{\text {eff }}$ which is then simply equal to the calculated effective thermal diffusivity $K_{\text {eff }}^{\prime}$ in the same model. In the general case, the actual effective thermal diffusivity in a composite is related to the effective thermal conductivity using the following equation that can be derived by inspection:

$$
K_{\text {eff }}=\frac{\lambda_{\text {eff }}}{\left(\rho C_{p}\right)_{\text {eff }}}
$$

where

$$
\left(\rho C_{p}\right)_{e f f}=\sum_{\text {all phases } i} \rho_{i} C_{p, i} g_{i}
$$

Examples of the Effective Thermal Diffusivity/Conductivity Calculated by the LMC method. In a model of a composite the LMC method for the calculation of the effective mass diffusivity (but without segregation) is thus exactly the same as the calculation of the effective thermal conductivity. Several recent LMC calculations of the effective thermal conductivity include calculations of the effective thermal conductivity of models of syntactic metallic hollow sphere structures (MHSS materials)[20] and compact heat sinks based on cellular metals[21,22]. An example of the results of a LMC calculation of the effective thermal conductivity is shown in Fig. 4 for the case of circular inclusions in a matrix[4]. In the same figure are also the results for the effective thermal conductivity using finite element analysis. The agreement is seen to be excellent.

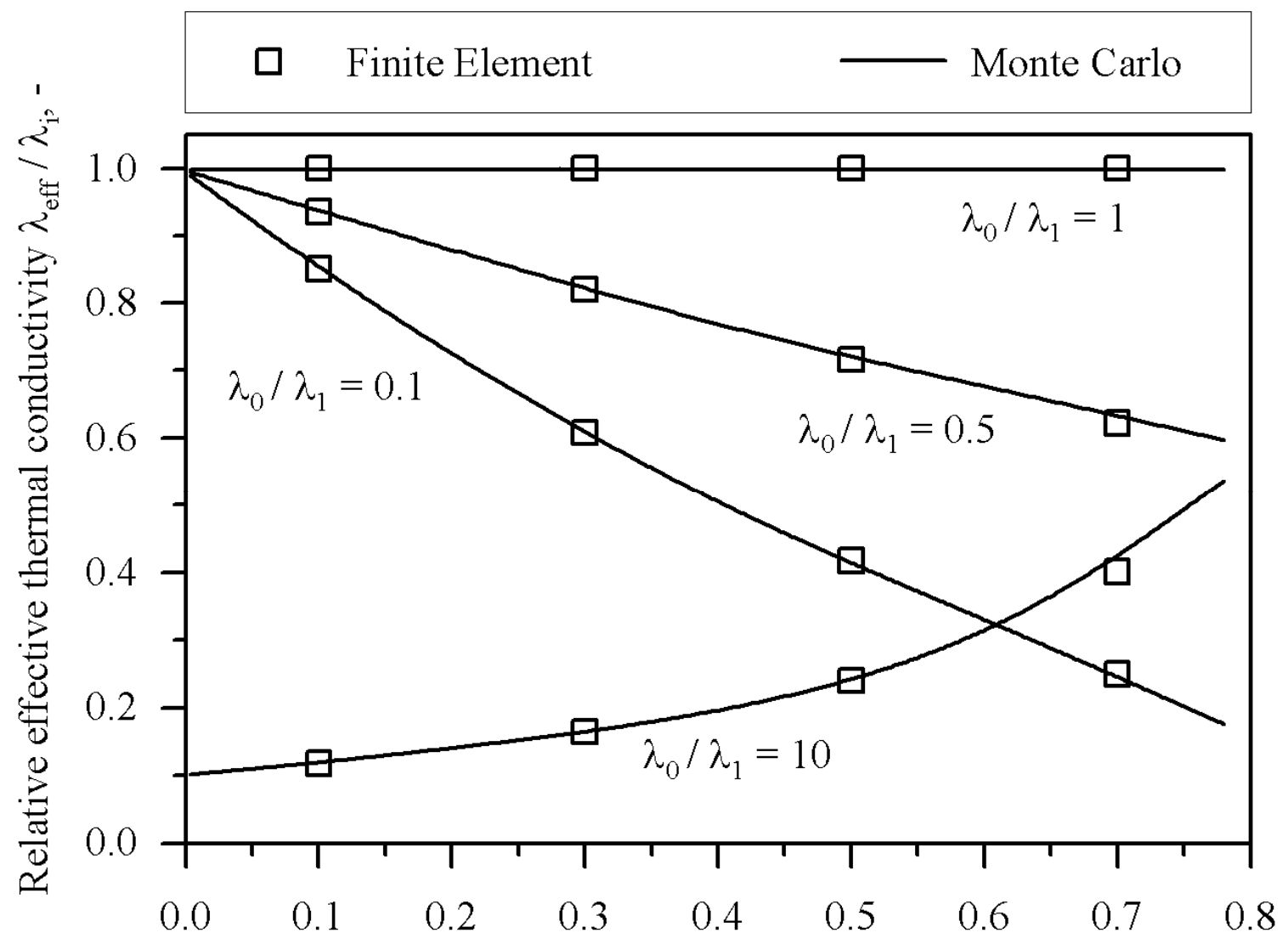

Area fraction of inclusions, $g$

Fig. 4. Comparison of results from LMC and Finite Element calculations of the relative effective thermal conductivity of a composite with circular inclusions (subscript 0 ) in a matrix (subscript 1) in a square planar arrangement as a function of area fraction of inclusions for several values of the matrix and dispersed phase thermal conductivities. 
The LMC Method for Determining Temperature Profiles. Obtaining the temperature profile using the LMC method is equivalent to solving the Heat Equation for the problem once initial and boundary conditions have been set. Because of the special role of the density and specific heat in transient thermal transport problems, the determination of temperature profiles requires a rather more involved procedure from that described for concentration profiles given above.

For the common situation where the surface temperature is held constant at $\mathrm{T}_{0}$, the number of virtual thermal particles at the source is held at $N_{\mathrm{n}}$. The results of the LMC analyses after some time $t$ are virtual thermal particle distributions. In order to obtain temperature profiles, the thermal particles are translated into site temperatures $T$ according to:

$$
T=\frac{n \cdot \mathrm{T}_{0} \rho \cdot C_{\mathrm{p}}}{N_{n} \cdot\left(\rho_{i} \cdot C_{\mathrm{p}, i}\right)_{\min }}=\frac{C_{\text {therm }} \cdot \mathrm{T}_{0} \rho \cdot C_{\mathrm{p}}}{\left(\rho_{i} \cdot C_{\mathrm{p}, i}\right)_{\min }},
$$

where $n$ is the number of virtual thermal particles currently located at the site in a phase with density $\rho$ and thermal capacity $C_{\mathrm{p}}$, (n relates to the concentration of the virtual thermal particles as $n$ $\left.=C_{\text {therm }} \cdot N_{n}\right)$; and $\left(\rho_{i} \cdot \mathrm{C}_{\mathrm{p}, \mathrm{i}}\right)_{\min }$ is the minimum value of that product over all phases. Further details can be found in ref [2].

Fig. 5 shows an example temperature profile obtained by LMC in a layered composite of aluminium and paraffin with the layers arranged normal to the heat flow. The thermal parameters of the two phases here are of course very different. In the same figure are the results of a determination of the temperature profile using finite element analysis. It can be seen that there is excellent agreement between these two methods.

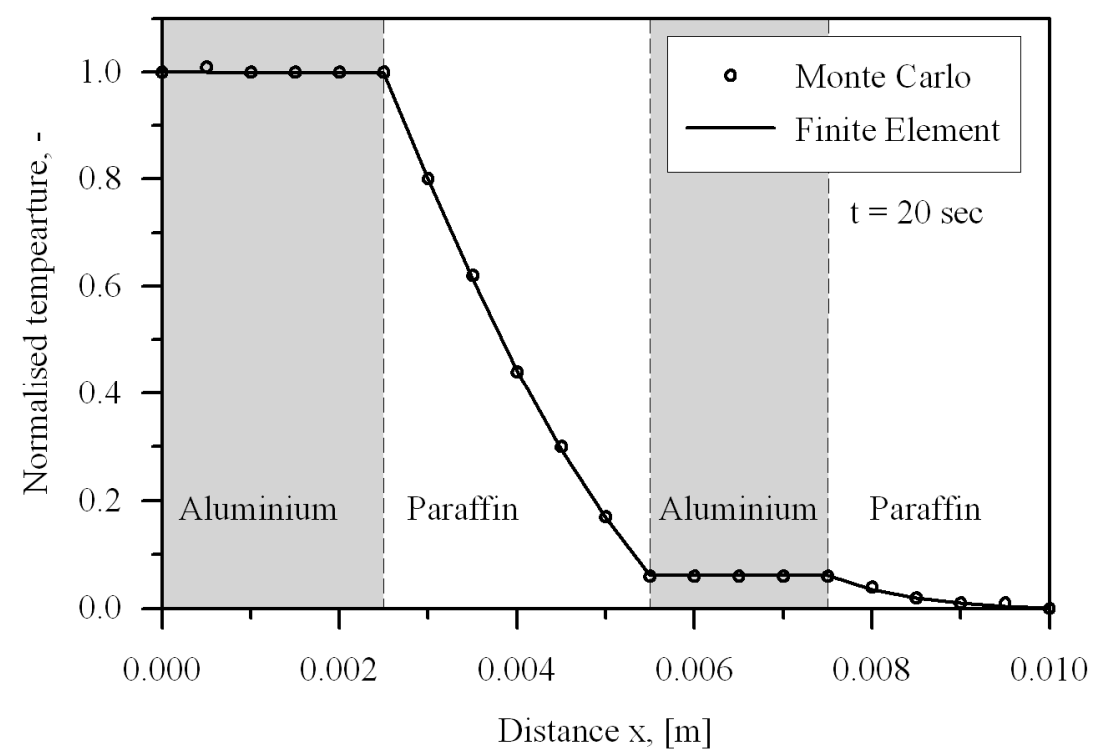

Fig. 5 The temperature profile determined by LMC and the finite element method for a layered aluminium - paraffin composite for a constant surface temperature.

\section{CONCLUSIONS}

In this overview, we introduced the recently developed Lattice Monte Carlo method for addressing and solving phenomenologically-based mass and thermal diffusion problems especially for composite materials. With examples, we describe the application of this numerical method to calculate effective mass diffusivities and concentration profiles. Next, we describe the application 
of this method to the calculation of effective thermal conductivities/thermal diffusivities and temperature profiles.

\section{ACKNOWLEDGEMENTS}

We wish to thank the Australian Research Council for its support of this work. One of us (T.F.) wishes to acknowledge the Portuguese Foundation of Science and Technology (FCT) for financial support.

\section{REFERENCES}

[ 1] I. V. Belova and G. E. Murch, in Cellular and Porous Materials: Thermal Properties, Simulation and Prediction, Wiley-VCH, Weinheim, (Eds.) Öchsner A, Murch G E and de Lemos J S, in press.

[ 2] I. V. Belova and G. E. Murch, Fiedler T and Öchsner A, Diffusion Fundamentals, in press.

[ 3] I. V. Belova and G. E. Murch, Solid St. Phenom., Vol. 129 (2007), p.1

[4] I. V. Belova, G. E. Murch, N. Muthubandara and A. Öchsner, Material Science Forum, Vol. 553 (2007), P. 51

[ 5] I. Kaur, Y. Mishin and W. Gust, in Fundamentals of Grain and Interphase Boundary Diffusion, Wiley, Chichester, (1995).

[ 6] P. Heitjans and S. Indris , J. Phys. Condens. Matter, Vol, 15 (2003), R1257

[ 7] I. Stloukal and J. Čermak, Defect Diffusion Forum, Vol. 263 (2007), p.189

[ 8] A. Einstein, Ann. Phys. (Leipzig), Vol. 17 (1905), p.549

[ 9] M. von Smolukowski , Ann. Phys. (Leipzig), Vol. 21 (1906), p.756

[10] H. Mehrer, in Diffusion in Solids, Springer, (2007).

[11] G. E. Murch , in Diffusion in Crystalline Solids, Academic Press, Orlando, (Eds) A. S. Nowick and G. E. Murch , (1984), p 379.

[12] I. V. Belova and G. E. Murch, in Mass and Charge Transport in Inorganic Materials, Techna, Fienza, (Eds.) Vincenzini and Buscaglia V, (2003) p 225.

[13] J. C. Maxwell-Garnett, Phil. Trans. Royal Soc., Vol. 203 (1904), p.386

[14] I. V. Belova and G. E. Murch, J. Meta. Nanocryst. Mat., Vol. 19 (2004), p. 23

[15] I. V. Belova and G. E. Murch, Phil. Mag., Vol. 84 (2004), p. 17

[16] I. V. Belova and G. E. Murch, J. Phys. Chem. Solids, Vol. 66 (2005), p. 722

[17] I. V. Belova and G. E. Murch, Phil. Mag. A, Vol. 81 (2001), p. 2447

[18] I. V. Belova and G. E. Murch, in Defect and Diffusion Forum, in press.

[19] I. V. Belova , N. Muthubandara , G. E. Murch , M. Stasiek and A. Oechsner , Solid State Phenomena, Solid. St. Phenom, Vol. 129 (2007), p. 111

[20] I. V. Belova and G. E. Murch, Journal of Materials Processing Technology, Vol. 153-154 (2004), p.741

[21] U. Ramamurty, A. Paul , Acta Mater., Vol. 52 (2004), p.869

[22] A. G. Evans , J. W. Hutchinson and M. F. Ashby, Prog. Mater. Sci., Vol. 43 (1999), p.171

[23] Nemat-Nasser, W. J. Kang, J. D. McGee , W. G. Guo and J. B. Isaacs , Int. J. Impact Eng., Vol. 34 (2007), p.1119 